\title{
Protótipo de Irrigação automatizada enquanto ferramenta para o ensino de computação: A Teoria na Prática
}

\author{
Julio Cezar da Silva Ferreira \\ Universidade Federal do Paraná \\ Palotina-Pr, Brasil \\ julio.silva@ufpr.br
}

\author{
Marcos Antonio Schreiner \\ Universidade Federal do Paraná \\ Palotina-Pr, Brasil \\ marcosantonio@ufpr.br
}

\author{
Paola Cavalheiro Ponciano \\ Universidade Federal do Paraná \\ Palotina-Pr, Brasil \\ paolaponciano@ufpr.br
}

\begin{abstract}
The costs with automated systems are generally high and inaccessible, for example, those in which the use of fresh water in irrigation for small producers is optimized and also those developed for educational purposes. In the last decades, the use of prototyping has been widely used in the school environment for teaching the most varied disciplines and even in an interdisciplinary way. Aiming at reducing costs with the implementation of an automation system, alternative means are used, such as prototyping with Arduino and scrap, thus, terms such as: Maker Culture and Project-Based Learning, have been increasingly used by researchers to explain and disseminate emerging teaching methodologies in the school environment. The present work is located in the confrontation between a proposal for the use of prototyping and the implementation of a real system for the automation of a grape vineyard at the Federal University of Paraná - Palotine Sector as a propelling tool in the teaching of Computing.

Keywords - Education; teaching; automation; prototyping.

Resumo - Os custos com sistemas automatizados geralmente são altos e inacessíveis, como por exemplo, aqueles em que se otimiza o uso de água doce nas irrigações para pequenos produtores e também aqueles desenvolvidos para fins educacionais. Nas últimas décadas, o uso de prototipagem tem sido muito utilizado no ambiente escolar para o ensino das mais variadas disciplinas e até mesmo de maneira interdisciplinar. Visando uma diminuição nos custos com a implantação de sistema de automação, utiliza-se meios alternativos, como a prototipagem com Arduino e sucata, com isso, termos como: Cultura Maker e Aprendizagem Baseada em projetos, tem sido cada vez mais utilizados por pesquisadores para explicar e divulgar as metodologias emergentes de ensino no ambiente escolar. $O$ presente trabalho situa-se no confronto entre uma proposta de uso de prototipagem e a implantação de um sistema real da automação de um parreiral de uva na Universidade Federal do Paraná - Setor Palotina como ferramenta propulsora do ensino de Computação.
\end{abstract}

Palavras-chave - Educação; ensino; automação; prototipagem.

\section{INTRODUÇÃO}

Este escrito enfatiza a integração entre as necessidades de automação da indústria e da agricultura com os processos de ensino de computação, por meio do desenvolvimento de um sistema de automação para o parreiral de uvas que encontra-se em andamento na Universidade Federal do Paraná - Setor Palotina.

O seu processo de desenvolvimento será utilizado como ferramenta de ensino da Ciência da Computação em um curso de Licenciatura em Computação e executado em parceria com o curso de Agronomia. Além de explorar o potencial enquanto ferramenta de ensino, o sistema de automação a ser implantado, tem a missão de diminuir o consumo de água, melhorar a qualidade das uvas e diminuir o trabalho repetitivo e braçal.

Da mesma forma, o projeto objetiva promover o ensino interdisciplinar da Ciência da Computação por meio do desenvolvimento do sistema automatizado de irrigação que atenda às necessidades pontuais da comunidade acadêmica, assim como atender programas de pesquisa e extensão futuramente. Tais ações serão desenvolvidas com estudantes matriculados nas disciplinas de Prática Pedagógica do Ensino em Computação I e Engenharia de Software do referido curso no ano letivo de 2021, usando a metodologia de aprendizagem baseada em projetos. Todo o desenvolvimento dos alunos será avaliado durante o processo de ensino.

O problema de pesquisa permeia o questionamento: é possível promover o ensino de ciência da Computação por meio do desenvolvimento de um projeto de irrigação automatizada de um parreiral de uvas?

\section{REFERENCIAL TEÓRICO}

\section{A. O Ensino de Computação}

A sociedade informacional impulsionada pelas Tecnologias de Informação e Comunicação demandam novas habilidades e competências na prática docente. No contexto deste trabalho trazemos o ensino de computação enquanto ciência, que conforme a Sociedade Brasileira de Computação [17] "possui fundamentos e princípios organizando de forma sistemática parte do conhecimento da humanidade. Computação pode ser considerada uma ciência natural: computação já existia muito antes de computadores (máquinas) serem inventados".

O ensino de computação atua como uma possibilidade de transformação do espaço de sala que usa conceitos da 
computação com fins educacionais utilizando como suporte o ensinar a ensinar e a promoção da interdisciplinaridade. De acordo com LÁZARO, SATO e TEZANI [9] os projetos interdisciplinares "[...] são elaborados em torno de um problema significativo para os alunos obterem um produto final, que é alcançado por meio de pesquisas em pequenos grupos".

Outrossim, as práticas inovadoras estão cada vez mais difundidas em sala de aula na contemporaneidade, tais estratégias se tornam necessárias para atender a uma demanda de mercado cada vez mais carente de pessoas com elevado poder de organização, de resolução de problemas complexos, de raciocínio lógico e trabalho em equipe capazes de atuar como protagonistas na construção do conhecimento e no desenvolvimento de novas tecnologias.

Tais práticas podem ser utilizadas a fim de proporcionar aos discentes um ambiente onde possam transformar o conhecimento em potenciais ferramentas de solucionar problemas no dia-a-dia. Conforme JESUS, SILVEIRA e PALANCH [8] "é preciso que o ambiente escolar não ensine estudantes apenas a memorizar conceitos, mas os ensine a lidar com problemas complexos de diferentes domínios, uma vez que o conhecimento está em constante evolução e as informações mudam e se expandem a cada dia".

Assim, o ensino de computação atua não somente na perspectiva de que o aluno seja passivo no processo de ensino aprendizagem, onde o estudante aprende a ser apenas usuário de tecnologias, mas também na perspectiva ativa em que possa criar, recriar, produzir e consolidar a produção de novas ideias e produtos usando ferramentas computacionais.

O graduando de Licenciatura em Computação fará um papel importante no processo de ensino aprendizagem. Estes profissionais inseridos no contexto escolar, terão a função fundamental de levar um rol de ideias de aulas criativas e inovadoras, com uso de tecnologias digitais, que podem instigar e atrair os alunos para o aprendizado e para o desenvolvimento dos saberes necessários aos profissionais da contemporaneidade.

Segundo GAROFALO [7], o docente contemporâneo “deve ter percepção e flexibilidade para assumir diferentes papéis: aprendiz, mediador, orientador e pesquisador na busca de novas práticas. Ele deve criar circunstâncias propícias às exigências desse novo ambiente de aprendizagem, assim como propor e mediar ações que levem a aprendizagem do aluno".

Nesta conjectura, o papel do educador na área de ensino de computação deve perpassar a ideia de que o aluno é um indivíduo suscetível de receber informações que serão úteis em algum momento da sua vida, aprendendo por meio de atividades que trabalhem conceitos da área de ciência da computação para além da tela do computador como no caso da cultura Maker e criação de protótipos, além do pensamento computacional e da robótica educacional, por exemplo.

No caso do projeto de irrigação automatizada, com Arduino e sensores, estas informações poderão servir para os acadêmicos do curso aprenderem conceitos técnicos da área de Ciência de Computação e também a aplicá-los de forma didática para ensinar os referidos conceitos.

\section{B. Pensamento Computacional}

No contexto do sistema de irrigação automatizada do parreiral de uvas, pode-se afirmar que o processo de desenvolvimento possibilita aos acadêmicos as experiências práticas para o entendimento dos conceitos teóricos relacionados à ciência da computação. Consequentemente, em se tratando do curso de Licenciatura em computação, a construção e a obtenção do produto final servem como meios de conhecer e compreender diferentes metodologias e procedimentos didáticos para o ensino destes conceitos, proporcionando a dualidade em que se aprende não somente a desenvolver mas também a melhor forma de ensinar a desenvolver.

Dentre as metodologias mais utilizadas neste contexto, está o Pensamento Computacional ou computational thinking que é uma forma que o indivíduo ou uma máquina se organiza de maneira similar a um algoritmo para resolver um problema, ou seja, resolver um problema por meio de uma sequência lógica de passos. Assim, BLIKSTEIN [3] sugere que o pensamento computacional é "saber usar o computador como um instrumento de aumento do poder cognitivo e operacional humano". O autor também ratifica que "Papert (1993) advoga a tecnologia nas escolas não como uma maneira de aperfeiçoar a instrução tradicional, mas como um conjunto de ferramentas emancipadoras que coloca nas mãos das crianças os materiais de construção mais poderosos". BLIKSTEIN [4].

O processo de construção de um sistema, pode instigar os pesquisadores, tanto docente quanto discente, a colocar em prática o uso do pensamento computacional para solucionar problemas antes mesmo de vivenciá-los na prática. No projeto de automação, por exemplo, essas situações surgem a todo momento, e cabe ao educador explorar estes problemas como potenciais recursos para sua aula.

\section{MATERIAIS E MÉTODOS}

Para iniciar o projeto, foi realizada a identificação dos requisitos do sistema bem como das possibilidades de execução de cada etapa necessária para a sua consolidação. Estas etapas consistem em: revisão da literatura, planejamento, desenvolvimento do sistema, testes e abordagem na perspectiva didática em sala de aula. Com relação aos requisitos, além do custo do projeto, manteve-se a preocupação em relação a durabilidade dos componentes que ficariam no campo, por exemplo, o sensor de umidade do solo que será um dos mais importantes no sistema de automação da irrigação do parreiral de uvas.

Nesta mesma fase, decidiu-se por utilizar a placa de prototipagem arduino para o módulo de controle do sistema, que é um hardware livre composto por microcontrolador e periféricos, sendo programável em código aberto. Esta placa é uma plataforma barata funcional e fácil de aprender, caracterizada como ideal para aprender e ensinar conceitos de diversas áreas do conhecimento quando utilizadas em projetos educacionais.

No decorrer das pesquisas conhecemos um sistema que foi desenvolvido por pesquisadores da Universidade Federal Rural do Rio de Janeiro (UFRR) denominado "pinga", que é um sensor de baixo custo e que não possui sistema elétrico nem metais em contato direto com o solo. "O sistema liga e desliga a irrigação em resposta à umidade do solo. Não há desperdício de água", BATISTA et al [2]. 
O funcionamento deste sensor de umidade acontece basicamente pela diferença de pressão entre um filtro de água e um pressostato. Esta diferença de pressão é possível mediante a variação de umidade do solo. A figura I, ilustra o sistema do sensor de umidade. funcionamento, por exemplo a fonte de alimentação dos componentes do painel de controle. Depois de analisar vários modelos de fontes de alimentação, foi escolhido o modelo de fonte ATX, por possuir várias saídas de tensão elétrica, atendendo assim às necessidades do projeto.

A tabela I, apresenta os materiais que serão utilizados durante o desenvolvimento do projeto de irrigação do parreiral de uva da Universidade Federal do Paraná - Setor Palotina.

TABELA I

TABELA DOS MATERIAIS

Fig. 1. "Pinga" - Acionador Simplificado para Irrigação[16].

Como é possível verificar na imagem, uma vez que o solo estiver seco ou com baixa umidade, o material poroso do filtro de água permite a passagem de ar da atmosfera e cria uma diferença de pressão suficiente para atuar o pressostato, que serve como um interruptor do sistema que ativa as válvulas solenoides liberando a água para irrigar o solo novamente.

Após estes estudos, foi realizada a escolha de um tema e uma problematização da temática no sentido de buscar abordagens didáticas que utilizam metodologias de ensino visando instigar nos discentes e docentes o desejo da busca pelo conhecimento sem precisar se "desligar" da realidade tecnológica da atualidade, promovendo o trabalho em equipe e a experimentação da teoria na prática.

No decorrer do desenvolvimento das atividades os discentes terão a oportunidade de testar os conceitos e as teorias aprendidas em sala de aula por intermédio da manipulação de objetos físicos que serviram como interface de entrada de dados, interpretadas pela lógica do sistema e gerando saídas de comandos elétricos para atuadores do sistema de irrigação relacionando a teoria e a prática.

\section{RESULTADOS PRELIMINARES}

Durante o desenvolvimento do projeto de automação, consultamos materiais, como controlador lógico programável (Controlador Lógico Programável - Modelo SS2 com 8 Entradas e 6 Saídas Digitais a Relé e Alimentação 24Vdc), que custava quase 28 vezes $(\mathrm{R} \$ 1005,00)$ a mais que um controlador de prototipagem, como o arduino (em torno de $\mathrm{R} \$ 35,00)$. Este foi um dos fatores que nos impulsionou a implantar um sistema alternativo que atendesse os interesses dos usuários do sistema, sem prejuízo ao sistema de automação e que tivesse o custo significativamente reduzido. Outro ponto importante a ser destacado, é em relação aos materiais que serão utilizados em campo, uma vez que alguns componentes e periféricos eletrônicos não suportam as variações climáticas e a ação do tempo em situações adversas.

Além do sensor de umidade do solo, outros pontos precisam de uma atenção especial por parte dos pesquisadores, uma vez que o sistema funcionará com comandos elétricos de baixa tensão e materiais eletrônicos sensíveis a ruídos elétricos.

Algumas das práticas que serão adotadas incluem, a sinalização do painel de comando e dos componentes instalados em campo, a fim de evitar risco de choque elétrico. Alguns materiais utilizados no sistema são de materiais que já foram descartados e que ainda estão em

\begin{tabular}{|c|c|c|}
\hline Item & Nome & Qtde \\
\hline 1 & Válvula Solenoide para Água 127V & 3 \\
\hline 2 & Arduino Uno R3 com cabo USB & 1 \\
\hline 3 & $\begin{array}{l}\text { Cabo Flexível Azul } 20 \text { AWG } \\
(0,50 \mathrm{mmX} 50 \mathrm{~m})\end{array}$ & 1 \\
\hline 4 & $\begin{array}{l}\text { Cabo Flexível Preto } 20 \text { AWG } \\
(0,50 \mathrm{mmX60m})\end{array}$ & 1 \\
\hline 5 & Chave Gangorra Verde com Neon & 3 \\
\hline 6 & Mangueira de nível 3/8'X5m & 1 \\
\hline 7 & Painel elétrico $280 \times 180 \times 140 \mathrm{~mm}$ & 1 \\
\hline 8 & Tubo de pvc $100 \mathrm{~mm} \times 1 \mathrm{~m}$ esgoto & 1 \\
\hline 9 & CAP $100 \mathrm{~mm}$ esgoto & 1 \\
\hline 10 & Borne porta-fusível $20 \mathrm{~mm}$ & 3 \\
\hline 11 & Disjuntor unipolar 2A & 1 \\
\hline 12 & Vela de filtro & 1 \\
\hline 13 & Fonte ATX & 1 \\
\hline 14 & Prensa cabo $1 / 2$ pvc & 2 \\
\hline 15 & Pressostato de máquina de lavar & 1 \\
\hline 16 & Adaptador mangueira com rosca 1' & 1 \\
\hline 17 & TE PVC roscavel 3/4 & 6 \\
\hline 18 & Joelho $3 / 4$ com rosca & 2 \\
\hline 19 & Registro $3 / 4$ pvc com rosca & 1 \\
\hline 20 & $\begin{array}{l}\text { Adaptador Pvc Solda Rosca Curto De } \\
\text { 25mm X 3/4 }\end{array}$ & 2 \\
\hline 21 & Condulete corrugado $1 / 2^{\prime}$ & 25 \\
\hline 22 & Módulo relé 4 canais $5 \mathrm{~V}$ & 2 \\
\hline
\end{tabular}

Durante a prototipagem, geralmente o sistema de controle funciona compartilhando os mesmos recursos elétricos do sistema de automação. Neste projeto, optou-se em separar a automação do controle. Este método foi necessário para garantir que o sistema de automação do parreiral funcione independentemente do sistema de controle.

Esta tática foi motivada pela falta de segurança no correto funcionamento dos componentes eletrônicos, que são altamente sensíveis a ruídos elétricos podendo comprometer o sistema. Desta maneira, a parte responsável pela automação da irrigação funcionará somente por comandos elétricos binários, interrompidos ou não pelo sinal do pressostato instalado em campo.

O arduino será responsável pela coleta das informações do processo, do solo e do tempo, a fim de gerar dados para tomadas de decisões pelo cliente em relação às plantas ou até mesmo o sistema. 
Os sensores de umidade são comumente encontrados para utilizar em conjunto com arduino e outros controladores, para simular leituras de umidades do solo em protótipos, porém os sensores disponíveis no mercado, foram testados e já comprovaram a sua baixa durabilidade, o que o torna impróprio para uso em campo por períodos longos de operação. Sua baixa durabilidade está diretamente ligada às condições climáticas adversas e o contato direto do seu material metálico com o solo, gerando assim a oxidação.

Percebe-se que ao longo do desenvolvimento do projeto e em contato com a situação real de operação do sistema, muitas variáveis devem ser consideradas (condições climáticas a quais os periféricos eletrônicos serão expostos, níveis de proteções contra surtos elétricos, situação do local de instalação do painel de comando entre outros), uma vez que estas podem significar prejuízos materiais, financeiros e até danos físicos para pessoas e animais que possam entrar em contato com aquele sistema.

Quando se trabalha com prototipação no ambiente de ensino, percebe-se que mesmo que um sistema automatizado não esteja funcionando perfeitamente conforme foi planejado, ainda é possível explorar didaticamente tudo o que é apresentado por aquele sistema, isto depende da expertise do docente em aproveitar os problemas e transformá-los em situações de aula.

\section{CONCLUSÃO}

No decorrer de parte do processo de automação da irrigação do parreiral de uvas já podemos perceber que o custo, a durabilidade dos materiais eletrônicos devem ser considerados para viabilizar o projeto de modo a atender as necessidades dos usuários. Deste modo, conceitos relacionados com as disciplinas de Engenharia de Software e Prática Pedagógica do Ensino de computação poderão ser transmitidos de modo a proporcionar ao aluno uma experiência de testar o conhecimento na prática.

Com relação à etapa de abordagens didáticas do projeto, espera-se que os estudantes consigam identificar a relação teoria e prática e as possíveis formas de ensinar.

\section{AGRADECIMENTOS}

Gostaríamos de agradecer ao espaço cedido pelo Professor Dr. Alessandro Jefferson Sato pelo espaço e oportunidade cedidos a nós para realização do projeto de automação por meio do projeto de produção de uvas. Agradecemos também ao ColabMaker da UFPR - Setor Palotina pelo incentivo a pesquisa de maneiras multidisciplinares e também a todos os cursos envolvidos e colaboradores que tornam possível o desenvolvimento de atividades que estimulam a vontade de aprender e pesquisar nos estudantes.

\section{REFERÊNCIAS}

[1] AGUIRRE, Luis Antonio. Enciclopédia de automática (vol. 3): controle e automação. Editora Blucher, 2007.

[2] BATISTA, Selma $\mathrm{CO}$ et al. Produção de alface regada automaticamente com controlador de baixo custo. Journal of Food, Agriculture \& Environment, v. 11, n. 2, pág. 485-489, 2013.

[3] BLIKSTEIN, Paulo. O pensamento computacional e a reinvenção do computador na educação. Education \& Courses, 2008. Disponível em $<$ http://www.blikstein.com/paulo/documents/online/ol_pensamento_co mputacional.html >. Acesso em: 26/09/2020.

[4] BLIKSTEIN, Paulo. Viagens em Troia com Freire: a tecnologia como um agente de emancipação. Educação e Pesquisa, v. 42, n. 3, p. 837856,2016

[5] FONSECA, S. M.; MATTAR, J. Metodologias ativas aplicadas à educação a distância: revisão da literatura. Revista EDAPECI, v. 17, n. 2, p. 185-197, 2017.

[6] FUHR, R. C.; HAUBENTHAL, W. R. Educação 4.0 e seus impactos no século xxi. "Educação no Século XXI-Volume 36 Tecnologia, p. 61, 2019.

[7] GAROFALO, Debora. Educação 4.0: o que devemos esperar. Nova Escola, 07 mar. 2018. Disponível em: $<$ https://novaescola.org.br/conteudo/9717/educacao-40-o-quedevemos-esperar $>$. Acesso em 10 set. 2020.

[8] JeSUS, A. M.; SILVEIRA, I. F.; PALANCH, W. B. de L. Desenvolvimento do pensamento computacional por meio da colaboração: uma revisão sistemática da literatura. Revista Brasileira de Informática na Educação, v. 27, n. 02, p. 69, 2019.

[9] LÁZARO, Adriana Cristina; SATO, Milena Aparecida Vendramini; TEZANI, Thaís Cristina Rodrigues. Metodologias ativas no ensino superior: o papel do docente no ensino presencial. CIET: EnPED, 2018.

[10] Libâneo, J. Carlos L. Democratização da escola pública, a pedagogia crítica social dos conteúdos. 6a ed. São Paulo: Loyola, 1984. Capítulo 1: Tendências pedagógicas na prática escolar, p.19-44.

[11] MORAES, Maria J. et al. Automação em sistema de irrigação tipo pivô central para economia de energia elétrica. Engenharia Agrícola, v. 34, n. 6, p. 1075-1088, 2014.

[12] Moran, J. M.. Mudando a educação com metodologias ativas. [Coleção Mídias Contemporâneas. Convergências Midiáticas, Educação e Cidadania: aproximações jovens. Vol. II] Carlos Alberto de Souza e Ofelia Elisa Torres Morales (orgs.). PG: Foca FotoPROEX/ UEPG, 2015. Disponível em: http://rh.unis.edu.br/wpcontent/uploads/sites/67/2016/06/Mudando-aEducacao-com-Metodologias-Ativas.pdf >. Acesso em: 08/10/2020.

[13] NUNES, D. J. Computação ou informática. Jornal da Ciência, v. 30, 2010.

[14] PAPERT, Seymour. The children's machine: Rethinking school in the age of the computer. BasicBooks, 10 East 53rd St., New York, NY 10022-5299, 1993.

[15] WING, Jeannette M. Computational thinking. Communications of the $A C M$, v. 49, n. 3, p. 33-35, 2006.

[16] WEBTRONICO. Acionador Simplificado para Irrigação. Disponível em:

$<$ http:/g1.globo.com/economia/agronegocios/globorural/noticia/2017/ 03/equipament o-ajuda-pequenos-agricultores-economizar-nairrigacao.html> Acesso em: 10/10/2020.

[17] Diretrizes para ensino de Computação na Educação Básica. Disponivel em: $<$ https://www.sbc.org.br/documentos-da-sbc/send/203-educacaobasica/1220-bncc-em-itinerario-informativo-computacao-2>. Acesso em: 18/11/2020. 